Journal of Animal and Veterinary Advances 11 (2): 207-210, 2012

ISSN: $1680-5593$

(C) Medwell Journals, 2012

\title{
Histopathological and PCR Mycobacterium bovis Identification in Mexican Opossums (Didelphis virginiana) from Hidalgo, Mexico
}

\author{
${ }^{1}$ R. Acosta-Salinas, ${ }^{1}$ J. Ocampo-Lopez, ${ }^{1}$ Z . Arellano-Balderrama, ${ }^{1}$ L. Ramirez-Perez, \\ ${ }^{1}$ V.M. Martinez-Juarez, ${ }^{1}$ J.I. Olave-Leyva, ${ }^{1}$ P.B. Garcia-Reyna, \\ ${ }^{2}$ G. Suzan-Azpiri and ${ }^{3}$ H.B. Barrios-Garcia \\ ${ }^{1}$ Area Academica de Medicina Veterinaria y Zootecnia, Instituto de Ciencias Agropecuarias, \\ Universidad Autonoma del Estado de Hidalgo, Mexico \\ ${ }^{2}$ Facultad de Medicina Veterinaria y Zootecnia, Universidad Nacional Autonoma de Mexico \\ ${ }^{3}$ Facultad de Medicina Veterinaria y Zootecnia, Universidad Autonoma de Tamaulipas, Mexico
}

\begin{abstract}
Mycobacterium bovis is the causal agent of bovine tuberculosis, this bacillus affects domestic wild animals as well as human beings, thus considered a zoonosis. Didelphis virginiana is the Mexican marsupial in spite of being a wild animal, it has been adapted to live near to human and it can be included in the people's diet. The opossum could be susceptible to be infected by $M$. bovis. There were captured six adult animals in the Mexican state of Hidalgo; the animals were sacrificed using an overdose of sodic pentobarbital; samples were taken from lung, spleen, intestinal tract, liver, mediastinic, retrofaringeal, submaxilar lymph nodes; Necropsy findings and histophatological lesions are suggestive of chronic disease as it occurs in tuberculosis cases. Histopatological study showed calcification perfectly surrounded by dense irregular collagen tissue in which a detachment of capsule is found. Two of the specimens in the study showed positive in tuberculosis, using nested PCR. The present study shows the risk that could exist during the transmission of tuberculosis maybe due to Didelphis virginiana coexistence with infected bovine cattle and human.
\end{abstract}

Key words: Didelphis virginiana, Mycobacterium bovis, $\mathrm{PCR}$, lung, disease, animals

\section{INTRODUCTION}

In Mexico, the bovine Tuberculosis (TB) is associated with both regionalization of the country, considering the levels of prevalence; in the state of Hidalgo, in the center of the country, the prevalence is $>0.2 \%$ in bovines. Mycobacterium bovis is the causal agent of bovine tuberculosis, this bacillus affects domestic as well as human beings, thus considered a zoonosis (Romero et al., 2006). The bacterium infects other animal species like wildlife and this range of hosts complicate attempts to control or eradicate the disease (Rodriguez et al., 2010).

Mexican opossum (Didelphis virginiana) is the only marsupial that inhabits the American continent (Hernandez-Huerta et al., 2002; Barrios-Garcia et al., 2009) which could be susceptible to be infected by $M$. bovis (Fitzgerald et al., 2003) and could represent a risk for some areas of exploitation of bovine (Witmer et al., 2010) due to that Mexican opossum cohabits around agricultural fields; it is important to mention that in this region of the country, opossum can be included in the people's daily diet, thus included in the gastronomical annually fair taken place in April at the municipality of Santiago of Anaya. This fair has an attendance of 15000 people average and around 1100 dishes are presented (Bautista, 2008; Barrios-Garcia et al., 2009). The aim of the current study was to analyze Mexican opossums as risk factor and transmitter of Mycobacterium bovis, identified through PCR and histopathology.

\section{MATERIALS AND METHODS}

Mexican opossum capture was in a zone near to domestic animals, especially bovine and sheep cattle as well as at grain harvest time such as corn and sorghum (September to December). Three sampling were done in Santiago Tulantepec and a total of six animals were caught, four females and two males with an average age of 2.6 years and average weight of $1100 \mathrm{~g}$. Captures were done using Tomahawk catch alive traps. Animals were sacrificed using an overdose of sodic pentobarbital

Corresponding Author: Hugo Brigido Barrios-Garcia, Facultad de Medicina Veterinaria y Zootecnia, Universidad Autonoma de Tamaulipas, Carr. Cd. Victoria-Mante Km 5, C.P. 87000 , Cd. Victoria Tamaulipas, Mexico 
(Anestesal ${ }^{\circledR}$ Pfizer). Pair samples were taken of each organ for histopathological and molecular analyses (lung, spleen, intestinal tract, liver, mediastinic, retrofaringeal, submaxilar lymph nodes), one was frozen at $-20^{\circ} \mathrm{C}$ and the other fixed in diluted formalin at 10\% (Prophet et al., 1995). For the histopathological study, the samples were processed by Paraffin-embedded method and $6 \mu \mathrm{m}$ sections were staining with Hematoxyilin-Eosin (H-E) and Ziehl-Neelsen (ZN) methods.

In order to check the presence of the $M$. tuberculosis complex by PCR, DNA extraction was done; the frozen samples were homogenized in $400 \mu \mathrm{L}$ of TE buffer with a polytron PT 1200E. The DNA extracting was made with lysozyme $\left(20 \mathrm{mg} \mathrm{mL}^{-1}\right)\left(1 \mathrm{~h}\right.$ at $\left.37^{\circ} \mathrm{C}\right), 10 \%$ Sodium Dodecyl Sulphate (SDS) and proteinase $\mathrm{K}\left(20 \mathrm{mg} \mathrm{mL}^{-1}\right)(10 \mathrm{~min}$ at $\left.65^{\circ} \mathrm{C}\right) ; 5 \mathrm{M} \mathrm{NaCl}, \mathrm{CTAB}$ solutions were incubated $10 \mathrm{~min}$ at $65^{\circ} \mathrm{C}$. The DNA purification was made using organic extraction with chloroform/isoamilic alcohol (24:1). DNA samples were precipitated with pure ethanol solution at $-20^{\circ} \mathrm{C}$ for precipitation. The mixture was then centrifuged at maximum rotations per minute for $10 \mathrm{~min}$. After centrifugation pure ethanol solution removed and DNA was provided with the application of $50 \mu \mathrm{L} \mathrm{TE}$ buffer. The purity and concentration of the DNA was quantified using the Nanodrop spectometer (Nanodrop technologies) (Estrada et al., 2004). The DNA integrity was determinated by electrophoresis observations. The amplification of the $372 \mathrm{pb}$ fragment of the MP70 gen of the $M$. tuberculosis complex were used for the tuberculosis diagnostic in the specimens (Romero et al., 2006). The nested PCR was made doing an initial PCR $25 \mu \mathrm{L}$ of final volume and of $15 \mathrm{mM}$ of TrisHCL (pH 8.05); $50 \mathrm{mM}$ of KCl; $200 \mathrm{mM}$ of each dNTPs; 2.5 $\mathrm{mMMgCl}_{2}, 0.4 \mu \mathrm{M}$ of the primers TB1F 5' GAA CAA TCC GGA GTT GAC AA 3' y TB1R 5' AGC ACG CTG TCA ATC ATG TA $3^{\prime}$ ( 30 cycle of $12^{\prime}$ to $96^{\circ} \mathrm{C}, 30^{\prime \prime} 94^{\circ} \mathrm{C}, 30^{\prime \prime}$ $55^{\circ} \mathrm{C}, 1^{\prime} 72^{\circ} \mathrm{C}$ ) and $500 \mathrm{ng}$ of DNA. A posterior reamplified of $1 \mu \mathrm{L}$ previous product with oligonucleotide M22-3, $5^{\prime}$ GCT GAC GCG TGC ACT GTC GGG C 3' and M22-4, 5' CGT TGG CCG GGC TGG TTT GGCC $3^{\prime}\left(94^{\circ} \mathrm{C} / 5^{\prime}, 35\right.$ cycle of $\left.94^{\circ} \mathrm{C} / 1^{\prime}, 65^{\circ} \mathrm{C} / 45^{\prime \prime}, 72^{\circ} \mathrm{C} / 42^{\prime \prime}, 72^{\circ} \mathrm{C} / 10^{\prime}\right)$. The amplified products were analyzed in Agar gel electrophoresis of $2 \%$ agarose gel with ethidium bromide.

\section{RESULTS AND DISCUSSION}

Animal physical conditions were good in general. The macroscopic lesions found at necropsy and the histopathological changes are shown in the Table 1. In one of the animals studied (Table 1), near $5 \mathrm{~mm}$ diameter whitish dots were observed in the dorsal surface of both lungs. At the mediastinal lymph nodes were observed granulomatous lesions mainly at cortical zone.
Table 1: Results by PCR and histopathology description of lesions in the necropsy of tuberculosis diagnostic in opossums

\begin{tabular}{llllr}
\hline $\begin{array}{l}\text { Opossums } \\
\text { identification }\end{array}$ & $\begin{array}{l}\text { Necropsy } \\
\text { observations }\end{array}$ & PCR & HEstopathological diagnostic \\
\hline 1 & NVL & - & $*$ & ZN \\
2 & NVL & - & $*$ & $*$ \\
3 & NVL & + & $\begin{array}{l}\text { Cronical } \\
\text { interstitial }\end{array}$ & - \\
& & & pneumonia & \\
4 & NVL & - & $*$ & $*$ \\
5 & Whitish dots in lungs, & + & Encapsulated & - \\
& supraclavicular lymph & & focal classification & \\
& nodes enlarged & & & - \\
\hline
\end{tabular}

The obtained results in the histopatological diagnostic in the opossum 3 years 5 are indication of TB. No Visible Lesion (NVL) were not made histopathology diagnostic because in the necropsy were no founded lesions that indicate TB $\left(^{*}\right)$

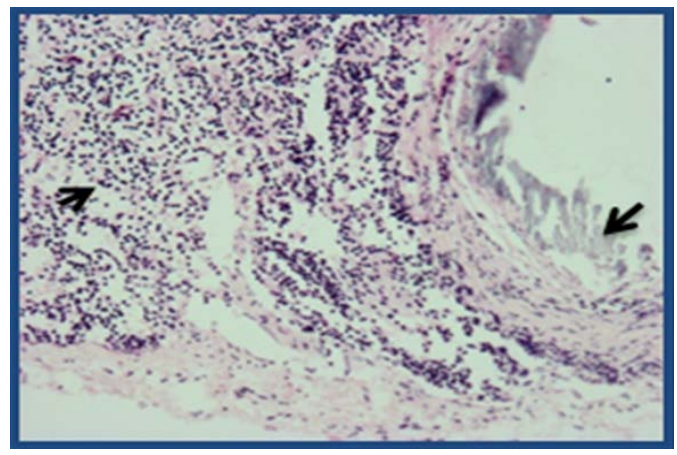

Fig. 1: Opossum five, Mediastinal lymph nodes showed encapsulated focal calcification surrounded by dense collagen tissue perfectly irregular and infiltration of macrophages, lymphocytes (left). $\mathrm{H}$ and $\mathrm{E}$ staining $100 \mathrm{X}$

At histopatological study with hematoxilin and eosin this animal showed calcification perfectly surrounded by dense irregular collagen tissue as consequence of swollen process in which a detachment of capsule is found. Those injuries could be a suggestion of chronic swollen process as in the tuberculosis case (Fig. 1, opossum 5), although when the Ziehl Neelsen staining no acid alcohol resistant bacillus were found.

Two of the specimens in the study showed positive in tuberculosis, using nested PCR, it was seen in it an amplification product of $208 \mathrm{pb}$ (Fig. 2). In Table 1, the obtained results by PCR, histopathology and the injury description to the necropsy to diagnose tuberculosis in opossums are shown. Meanwhile the rest of the studied specimens do not show amplifications to $M P 70$ gen. The summary of results is shown in Table 1.

Necropsy findings and histophatological lesions are suggestive of chronic disease as it occurs in tuberculosis cases. It is important to consider the bovine tuberculosis in the state of Hidalgo in its control stage. A factor that is affecting the bovine tuberculosis control programs 


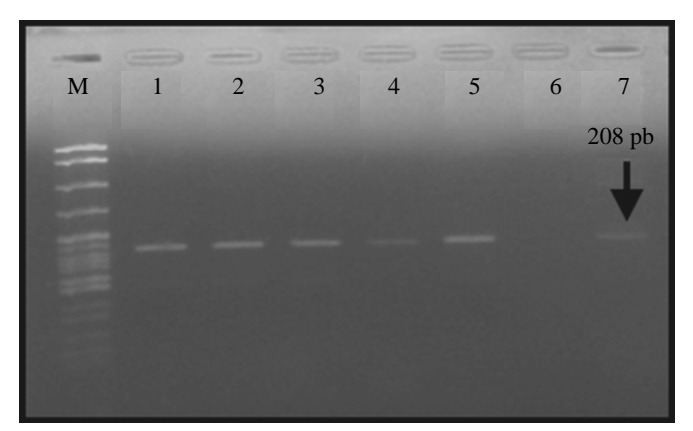

Fig. 2: Results of nested PCR shown positive specimens. M: Molecular weight marker. In the line 1: correspond to opossum number 3: Lung; line 2: lymph node, opossum 5: line 3: Lung; line 4: Mediastinal lymph node; line 5: Retropharyngeal node; line 6: negative control (Water MILI-Q) that corroborated specificity of the test; line 7: Positive control (stock AN5 DNA)

could be the wildlife presence, since they are not subject to governmental control programs as cattle (Woodroffe et al., 2006; Witmer et al., 2010). Thus, it could be a source of the disease spreading to healthy heads because they are usually feed close to farm animals feeders which makes them liable to get infected of bovine tuberculosis and remain as reservoir of the disease and then to be able to transmit it to humans, since these wildlife are part of human diet. On the other hand, Corner (2006) reported that $M$. bovis detected in cattle and deer was found in wildlife animals (Opossums, deers, pigs) and they concluded that genomic track of isolated $M$. bovis was the same as in bovine cattle. It has been reported that there are two types of susceptible animals to the infection by $M$. bovis, those that act as reservoir host or those which maintain or spread the disease called spillover animals could get infected but occurrence of disease is low among the population of these animals may be due to that the tuberculosis is slow, progressive and that individuals may possess resistance to the disease and only persists inside the population when there is a reservoir host in the bovine tuberculosis ecosystem (Thoen et al., 2006). Diegel et al. (2002) has been reported in previously studies that animals could develop tuberculosis. There are two very important factors to be considered: Transmission track and inoculated dose necessary to develop the disease but clinically animals may not have symptoms or during necropsy granulomatous injuries could be found which are characteristic of the disease or absence of it. This researcher in experimental studies has observed that the opossum is possible to get infected by $M$. bovis, a dose of $1 \times 10^{7} \mathrm{cfu}^{-1}$ using nasal track. Other studies proved oral and intramuscular track with a dose of $1 \times 10^{5} \mathrm{cfu} \mathrm{mL}^{-1}$ of the bacillus. In the case of inoculation VO of 4 inoculated, only one gave positive to culture (Intestine and lymph nodes) while those inoculated IM, 3 of them gave positive to culture (Lymph nodes, liver and kidney); only one animal gave negative to culture in spite of having the characteristic injuries of tuberculosis. This suggests that opossums ( $D$. virginiana) were sensible to infection by $M$. bovis by different tracks which occur in a natural way. Nevertheless, their susceptibility is relative; its importance is in acting as spreading species spillover (Fitzgerald et al., 2003).

PCR technique has been used now a days as a rapid diagnostic technique and highly sensible compared with the culture techniques. Nested PCR detects even fentograms (Estrada et al., 2004), besides its high specificity and that only detects to $M$. tuberculosis species including $M$. bovis; this characteristic makes this technique ideal for tuberculosis diagnosis. It is probable that due to this high sensibility a larger number of positive animals have been identified compared to the histopathological technique (Table 1). From these results, the researchers concluded that opossums are getting infected with the bacillus as shown by PCR tests, may be due to coexistence with infected bovine cattle, it is possible that infective dose needed to cause macroscopic and microscopic lesions observable in these animals could not be reached. In the case of the only animal with such suggesting lesions they could be due to constant reinfections by domestic animals; this coincides with reports where in spite of having identified the opossum as a species susceptible to get infected by $M$. bovis such susceptibility is low compared with host reservoirs, thus this will be catalogued as spreading species that only get infected when tuberculosis incidence when its habitat is high, it can return infected by this way and may transmit infection to other animals of its same species as well as to cattle and human beings (Thoen et al., 2006).

It would be interesting to study the state of infection in Mexican opossum in regions with a higher incidence of bovine tuberculosis to be able to evaluate if there is a higher number of infected animals and if they could really be a risk to public health of inhabitants of the state of Hidalgo who consume such animals in their diet.

\section{CONCLUSION}

With this study, the researchers have observed that a third of the studied animals were found infected by tuberculosis, even though it has have been reported that infective dose do not reach the necessary level to spread the disease amongst other animals, thus the researchers can not consider as disease reservoirs for the regional 
bovines, even though we can not rule out that they represent a public health issue, since the researchers know that they are part of local diet in some communities in this state.

\section{ACKNOWLEDGEMENTS}

Lic. Claudia Almazan-Garcia by her help in proofreading this research. Barrios-Garcia HB received support from the project PROMEP/UAT-PTC-122; support was also provided through proyect FOMIXTAMPS-2008-C17-107247. Acosta-Salinas R received support for F-PROMEP-38/Rev-03, Sep-23-005.

\section{REFERENCES}

Barrios-Garcia, H.B., R. Acosta-Salinas, J.P. AcostaDibarrat, P.B. Garcia-Reyba and V.M. MartinezJuarez, 2009. Antibiotic susceptibility of bacteria isolated from virginia opossum (Didelphis virginiana) in hidalgo, Mexico. J. Anim. Vet. Adv., 8: 2075-2078.

Bautista, U.J., 2008. Physical-chemical and nutritional quality of skunk meat. B.A. Thesis, Universidad Autonoma del Estado de Hidalgo, Instituto de Ciencias Agropecuarias.

Corner, L.A., 2006. The role of wild animal populations in the epidemiology or tuberculosis in domestic animals: How to assess the risk. Vet. Microbiol., 112: 303-312.

Diegel, K.L., S.D. Fitzgerald, D.E. Berry, S.V. Church, W.M. Reed, J.G. Sikarskie and J.B. Kaneene, 2002. Experimental inoculation of North American opossum (Didelphis virginiana) with mycobacterium bovis. J. Wildl. Dis., 38: 275-281.
Estrada, C.C., O.F. Diaz, D.C. Arriaga, N.S. Villegas, G.R. Perez, D. Gonzalez and S.D. Gonzalez, 2004. PCR Concordance and routine methods for bovine tuberculosis diagnostics. Vet. Méx., 35: 225-236.

Fitzgerald, S.D., L.S. Zwick, K.L. Diegel, D.E. Berry and S.V. Church et al., 2003. Experimental aerosol inoculation of mycobacterium bovis in North American opossums (Didelphis virginiana). J. Wildl Dis., 39: 418-423.

Hernandez-Huerta, A., C. Delfin-Alfonso and O. Munoz, 2002. Range extension of the opossum (Didelphis virginiana) in the Chihuahuan desert of Mexico. Southwestern Naturalist, 47: 127-129.

Prophet, B.E., B. Mills, B.J. Arrington and H.L. Sobin, 1995. Hystotechnological Methods. Pathology Institute of American Armed Forces, Washington, D.C.

Rodriguez, S., B. Romero, J. Bezos, L. de Juan and J. Alvarez et al., 2010. High spoligotype diversity within a Mycobacterium bovis population: Clues to understanding the demography of the pathogen in Europe. Vet. Microbiol., 141: 89-95.

Romero, T.A., D.C. Arriaga, V.J. Guevara, S.J.A. Garcia, L.R.A. Torres and C. Estrada-Chavez, 2006. Confirmation of Mycobacterium bovis excretion in nasal exudates using nested PCR in a dairy cattle herd. Vet. Mex., 37: 137-143.

Thoen, C., P. Lobue and I. de Kantor, 2006. The importance of Mycobacterium bovis as a zoonosis. Vet. Microbiol., 112: 339-345.

Witmer, G., A.E. Fine, J. Gionfriddo, M. Pipas, K. Shively, K. Piccolo and P. Burke, 2010. Epizootiologic survey of Mycobacterium bovis in wildlife and farm environments in Northern Michigan. J. Wildl. Dis., 46: $368-378$.

Woodroffe, R., C.A. Donnelly, H.E. Jenkins, W.T. Johnston and D.R. Cox et al., 2006. Culling and cattle controls influence tuberculosis risk for badgers. Proc. National Acad. Sci., 103: 14713-1 4717. 